This is the peer-reviewed version of the paper:

Pejovic-Milovancevic, M.; Grujicic, R.; Stojkovic, A.; Radosavljev-Kircanski, J. Changes in Hospital Treatment of Children and Adolescents with Mental Health Problems. Gen. Hosp. Psychiatry 2020, 64, 108-109. https://doi.org/10.1016/j.genhosppsych.2019.05.003.

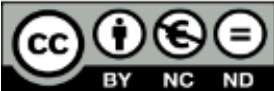

This work is licensed under the Attribution-NonCommercial-NoDerivatives 4.0 International (CC BYNC-ND 4.0) 


\section{Changes in hospital treatment of children and adolescents with mental health problems}

\section{Introduction}

Inpatient treatment is the most complex and expensive form of psychiatric treatment but sometimes is unavoidable. Deciding on the necessity of such treatment is not an easy step when it concerns children and adolescents (CA), and often requires an evaluation by a team of experts $[1,2]$. We aimed to determine whether there were any changes in the nature or duration of this important treatment over a two decade period at a child and adolescent inpatient unit in Serbia.

\section{Methods}

In this retrospective study, the database comprised 3977 patients aged 0 to $18(\mathrm{M}=11.8$ $\mathrm{SD}=4.7), 55.3 \%$ of whom were male, who were hospitalized at the Clinic for Children and Youth at the Institute of Mental Health in Belgrade, Serbia between 1996 and 2015. The data collected included: the number of patients per year, patient age, frequency of hospital treatment per patient $(\mathrm{M}=1.7 ; \mathrm{SD}=1.9)$, length of hospital stay $(\mathrm{M}=21.6, \mathrm{SD}=18.2)$ and the diagnoses. We used Pearson correlations to examine the relationships between variables and number of years from the initial assessment (1996), and independent samples $t$-tests to compare duration of hospitalization in boys and girls. This study was approved by the Ethics board of the Institute of Mental Health.

\section{Results}

Table 1 shows the frequency of diagnosis categories. The largest diagnostic category (22.6\%) was the category of disorders of psychological development (which includes: language disorders, learning disorders, motor disorders and autism spectrum disorders), followed by those within the categories of behavioral and emotional disorders (20.0\%) and mental retardation $(12.9 \%)$. The results show that the number of patients increased with time $(r=0.77$; $p<0.01)$. The upward trend in the number of patients was followed by the upward trend in the number of diagnoses per patient $(r=0.20 ; p<0.01)$.

The hospital stay decreased over time $(r=-0.09 ; p<0.01)$. There was a negative correlation between the year of admission and both the age of the patient $(r=-0.08 ; p<0.01)$ and the length of hospital stay $(r=0.45 ; \mathrm{p}<0.01)$. This demonstrates that during the course of time, younger patients were hospitalized more frequently for shorter periods, and also are rehospitalized more often $(r=-0.05 ; p<0.01)$. As expected, negative correlation $(r=-0.17 ; \mathrm{p}<$ 0.01) was found between the length of hospital stay and the frequency of hospitalization. Patients who returned for hospital treatment again had shorter admissions at the Department. Notably, female patients stayed longer in hospital $(M=23.9)$ than male patients $(M=19.8)$ $(t=6.4 ; p<0.01)$.

\section{Discussion}

A trend of increasing hospitalizations was observed over this 20 year period. This is consistent with the overall risk in psychiatric disorders in children, both in Serbia and globally [3-5]. In contrast, the capacities for inpatient treatment and staffing have been almost the same in the country over the last two decades. If this trend continues to rise, policymakers should consider increasing the current hospital resources (medical and non-medical staff, number of 
beds) so that the services can continue to provide support and help for children and adolescents that need it.

Although the number of hospitalizations has increased, our results demonstrate that the length of hospital stay has shortened over time. Moreover, the current trend of using valid diagnostic tests, applicable treatment guidelines, and effective team co-ordination, both within the institutions and with other sectors (schools, social services, other healthcare institutions) may have reduced the need for longer stays through higher quality of care [6].

Younger patients are hospitalized more frequently for shorter periods of time because they are usually admitted for diagnostic processing and treatment of comorbidities, while older patients, in addition to diagnostic procedure, are involved in various treatment procedures that require a longer period. One potential cause for a greater number of rehospitalizations was the trend toward shorter admissions, and some patients may not have been fully stabilized by discharge.

Readmissions in younger children are not surprising, given that there are few outpatient services for such children, and diagnostic evaluations and additional services that might occur in outpatient settings must occur in the hospital. For adolescents, longer admissions may actually be beneficial in the long run, and other forms of hospitalization, such as day hospitalization, are more preferable. Research shows that one of the key predictors of a longer hospital stay is impulsiveness and potential for self-injury [7], with a much greater prevalence among girls [8].

This may explain the longer admissions for girls, as patients with more behavioral dysregulation and self-injury may require additional time for stabilization compared to other patient groups. These findings suggest that additional outpatient mental health treatment services are needed, as in many cases the volume of admissions is too high for current staffing levels, and some treatment services (especially for young children) could be provided in the outpatient setting, preventing the need for admission.

\section{References}

[1] Blanz B, Schmidt MH. Preconditions and outcome of inpatient treatment in child and adolescent psychiatry. J Child Psychol Psychiatry 2000;41(6):703-12.

https://doi.org/10.1111/1469-7610.00658

[2] Lee Y, Martin P, Hembry P, Lewis S. Outcomes of inpatient psychiatric treatment for adolescents: a multiple perspectives evaluation. Clin Child Psychol Psychiatry

2017;1:135910451773907.

[3] Case BG, Olfson M, Marcus SC, Siegel C. Trends in the inpatient mental health treatment of children and adolescents in US community hospitals between 1990 and 2000. Arch Gen Psychiatry 2007;64(1):89-96.

https://doi.org/10.1001/archpsyc.64.1.89

[4] Blader JC. Acute inpatient care for psychiatric disorders in the United States, 1996 through 2007. Arch Gen Psychiatry 2011;68(12):1276-83.

https://doi.org/10.1001/archgenpsychiatry.2011.84

[5] Zima BT, Rodean J, Hall M, Bardach NS, Coker TR, Berry JG. Psychiatric disorders and trends in resource use in pediatric hospitals. Pediatrics2016;138(5):e20160909

https://doi.org/10.1542/peds.2016-0909 
[6] Indig D, Gear C, York A. The role of inpatient care for children and adolescents with moderate-to-severe mental disorders: an evidence check rapid review brokered by the Sax Institute (www.saxinstitute.org.au) for the NSW Ministry of Health. 2017.

[7] Ithman MH, Goplarkrishna G, Beck NC, Das J, Petroski G. Predictors of length of stay in an acute psychiatric hospital. J Biosafety Health Educ 2014;2:119.

[8] Monto MA, McRee N, Deryck FS. Nonsuicidal self-injury among a representativ 